\title{
Detection of IgG and IgA against the mycobacterial antigen A60 in patients with extrapulmonary tuberculosis
}

Marco Alifano, Roberto De Pascalis, Matteo Sofia, Stanislao Faraone, Mariassunta Del Pezzo, Italo Covelli

\begin{abstract}
Background-Diagnosis of extrapulmonary tuberculosis is often difficult to establish using standard methods. Serological techniques based on detection of antibodies against mycobacterial antigen A60 have shown good sensitivity and specificity in pulmonary tuberculosis. The present study was undertaken to define the diagnostic accuracy of testing for IgG and IgA against A60 in extrapulmonary tuberculosis.
\end{abstract}

Methods-One hundred and ninety eight subjects were studied: 42 patients with extrapulmonary tuberculosis confirmed by microbiology and/or histology, 24 subjects with healed pulmonary or extrapulmonary tuberculosis, 44 patients with a defined non-tuberculous disease, and 88 healthy volunteers (44 PPD negative and 44 PPD positive). Detection of IgG and IgA against $A 60$ antigen was carried out by enzyme-linked immunosorbent assay. Cut off values were determined by receiver operating characteristic curves.

Institute of Respiratory Disease

M Alifano

M Sofia

S Faraone

Institute of Clinical

Microbiology,

Department of

Cellular and Molecular

Biology and Pathology

$\mathrm{R}$ De Pascalis

M Del Pezzo

I Covelli

University of Naples "Federico II", Naples, Italy

Correspondence to: Dr M Alifano, Via Roma 60, I-81024 Maddaloni, Italy.

Received 23 July 1997

Returned to authors

5 September 1997

Revised version received

4 December 1997

Accepted for publication

21 January 1998 $73.8 \%$ in extrapulmonary tuberculosis, while the specificity was $96.1 \%$. The IgA test showed a sensitivity of $69.0 \%$ with a specificity of $93.6 \%$. Combination of the IgG and IgA tests showed a sensitivity of $80.9 \%$ and a specificity of $92.3 \%$. Patients with extrapulmonary tuberculosis showed significantly higher titres of both IgG and IgA against $A 60$ than other groups. Conclusions-Anti-A60 IgG or IgA tests are characterised by good sensitivity and specificity. The combined use of both tests allows an increase in diagnostic accuracy of extrapulmonary tuberculosis.

(Thorax 1998;53:377-380)

Keywords: extrapulmonary tuberculosis; serodiagnosis; antigen 60

Extrapulmonary tuberculosis accounts for approximately one third of all cases of tuberculosis. ${ }^{12}$ Diagnosis is easily established when pulmonary tuberculosis is associated.
However, this event occurs in only approximately $20 \%$ of cases. ${ }^{1}$

Detection of acid-fast bacilli on samples from the affected tissue (or fluid) is a rapid and inexpensive method. Sensitivity of less than $50 \%$ is reported ${ }^{1}$ and, in particular, only $37 \%$ of cases of meningeal tuberculosis are smear positive. $^{3}$

Detection of Mycobacterium tuberculosis in culture and/or characteristic histological features are generally required to establish the diagnosis. Sensitivity of these methods is moderately good $(71 \%$ according to Weir and Thornton $\left.{ }^{1}\right)$. However, invasive procedures are often required to obtain samples and the growth time of $M$ tuberculosis is unacceptably long. Other causes of granuloma formation may cause false positive results on histological examination.

A remarkable diagnostic delay (median two months) is frequent in extrapulmonary tuberculosis, ${ }^{1}$ though it is well known that any delay may be the cause of significant morbidity or mortality, especially in the central nervous system or pericardial tuberculosis.

Methods based on amplification of myco- bacterial DNA by the PCR technique are both expensive and laborious and specimens for analysis must often be taken by invasive procedures; their sensitivity and specificity are not absolute. $^{45}$

Several serological methods have been proposed, both in the past decades and more recently for the diagnosis of pulmonary tuberculosis. ${ }^{6-8}$ They are characterised by simplicity and rapidity of technical execution as well as financial viability. Searching for $\operatorname{IgG}^{9} 10$ and $\operatorname{IgA}^{11}$ against the antigen 60 (A60), the main thermostable component of Old Tuberculin and Purified Protein Derivative (PPD), provided satisfactory results in terms of sensitivity and specificity in the diagnosis of pulmonary tuberculosis.

The usefulness of A60-based serological tests in the diagnosis of extrapulmonary tuberculosis has been less extensively evaluated. Satisfactory characteristics of diagnostic accuracy have been reported in a study undertaken in India by Gupta et al. ${ }^{12}$ 
Table 1 Characteristics of groups studied

\begin{tabular}{lllll}
\hline Groups & No. of cases & No. of men & $\begin{array}{l}\text { Mean }(\mathrm{SD}) \text { age } \\
\text { (years) }\end{array}$ & No. PPD positive \\
\hline HC PPD negative & 44 & 20 & $41.2(11.3)$ & 0 \\
HC PPD positive & 44 & 25 & $40.1(11.7)$ & 44 \\
N-TB & 44 & 23 & $42.5(10.6)$ & 5 \\
hTB & 24 & 11 & $46.5(8.6)$ & 23 \\
EpTB & 42 & 18 & $41.4(13.7)$ & 20 \\
\hline HC = healthy controls; N-TB = non-tuberculous disease; hTB = healed pulmonary (n=15) or \\
extrapulmonary (n=9) tuberculosis; EpTB = extrapulmonary tuberculosis.
\end{tabular}

In the present work we have evaluated the diagnostic usefulness of measuring anti-A60 IgG and IgA levels in patients with extrapulmonary tuberculosis from a western country.

\section{Methods}

SUBJECTS

One hundred and ninety eight subjects were recruited (table 1). All were Caucasian living in Southern Italy. All were HIV negative and previously non-vaccinated with BCG (BCG vaccination is mandatory in Italy only for some professional categories). None of the patients had been treated with oral or parenteral steroids in the preceding six months.

Forty two patients with extrapulmonary tuberculosis confirmed by histological testing (evidence of caseating granulomas) or microbiology (cultural isolation of $M$ tuberculosis) were studied. The sites of extrapulmonary tuberculosis were lymph nodes $(\mathrm{n}=15)$, bones and joints $(n=10)$, genitourinary tract $(n=7)$, meninges $(n=4)$, pericardium $(n=2)$, adrenal glands $(n=2)$, larynx $(n=1)$, thyroid $(n=1)$. None had clinical or radiological evidence of concurrent active pulmonary tuberculosis. Clinical signs (localised and/or systemic) of active disease were present in all the cases for 10-46 days (median 24). No patient had received anti-tuberculous treatment.

The following subjects acted as controls: (1) 88 healthy volunteers (44 PPD positive and 44 PPD negative) with no signs of clinical impairment and normal chest radiographs who were recruited from a population of 620 blood donors; (2) 44 patients admitted to hospital for a defined acute or chronic non-tuberculous disease (five with bronchial asthma, six with acute bacterial pneumonia, 12 with infective exacerbation of COPD, nine with lung cancer, two with idiopathic lung fibrosis, two with heart failure, two with viral infection, two with rheumatoid arthritis, two with urinary tract infection, two with sarcoidosis); (3) 24 subjects treated for active pulmonary tuberculosis $(\mathrm{n}=$ 15) or extrapulmonary tuberculosis $(n=9)$ in the past 7-11 years and with no signs of disease reactivation in the preceding six years.

\section{PPD TESTING}

PPD testing was carried out by intracutaneous injection of $0.1 \mathrm{ml}$ of $5 \mathrm{TU}$ PPD (Biocine, Siena, Italy). An area of induration with a diameter of $\geqslant 10 \mathrm{~mm}$ at 48 hours was judged to be a positive response.

DETECTION OF ANTIBODIES AGAINST A60

Measurement of IgG and IgA against A60 was performed according to a standard protocol ${ }^{11}$
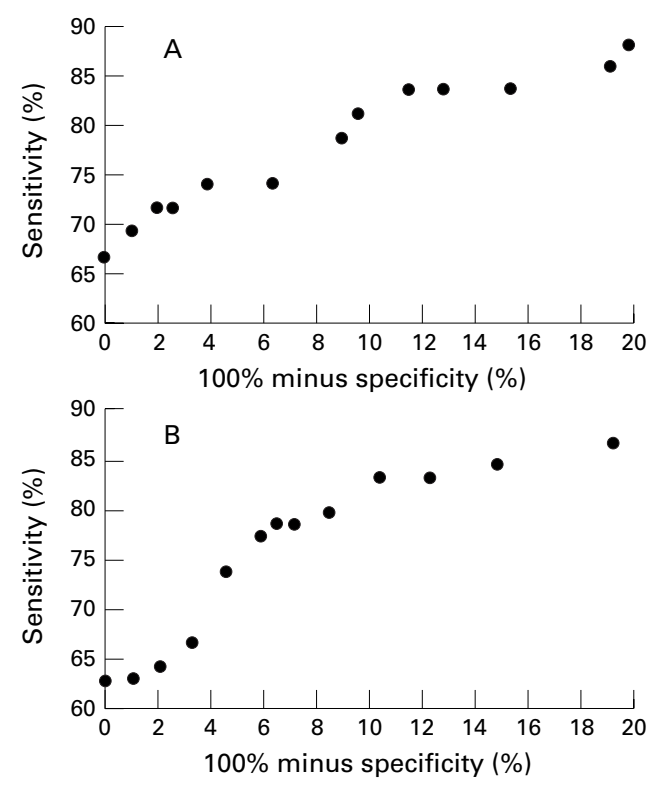

Figure 1 Receiver Operating Characteristic (ROC) curve of $(A)$ the IgG test and (B) the IgA test. Each point on the curves indicates the true positive rate (sensitivity) and the false positive rate (100\% minus specificity) for a particular cut off value (optical density for the IgG test, 0.281-0.428; optical density for the IgA test, 0.250-0.447).

by the ELISA method using commercially available kits (Anda Biological, Strasbourg, France).

\section{DATA ANALYSIS}

Demographic data of subjects from different groups were compared by the one-way analysis of variance test. Results of determinations of antibody titres were compared by Wilcoxon's rank sum test. Statistical significance was accepted at a level of $\mathrm{p}<0.05$.

Cut off values were established by the receiver operating characteristic (ROC) curve technique, ${ }^{13}$ a method widely employed in the medical decision making sequence, especially in both radiology and nuclear medicine. To draw ROC curves true positive rates (sensitivity) and false positive rates $(100 \%$ minus specificity) were calculated at different cut off values and plotted on a graph (fig 1). The ROC curves describe the relationships between the probabilities of correct and incorrect results at different cut off values. The points that enclose the largest area or, less formally, that lie farthest to the "northwest" of the graphs were believed to be the most accurate cut off values ${ }^{14}$ and were chosen for this purpose.

\section{Results}

No difference was found with regard to the sex and age of the subjects in the different groups.

IgG test

The results of measuring IgG against A60 are displayed in fig 2A. Patients with extrapulmonary tuberculosis showed significantly $(p<0.0001)$ higher titres of anti-A60 IgG than control groups. With the cut off value of 0.371 OD, established according to the ROC method (see above), the test was positive in 31 of 42 patients with extrapulmonary tuberculosis. For 

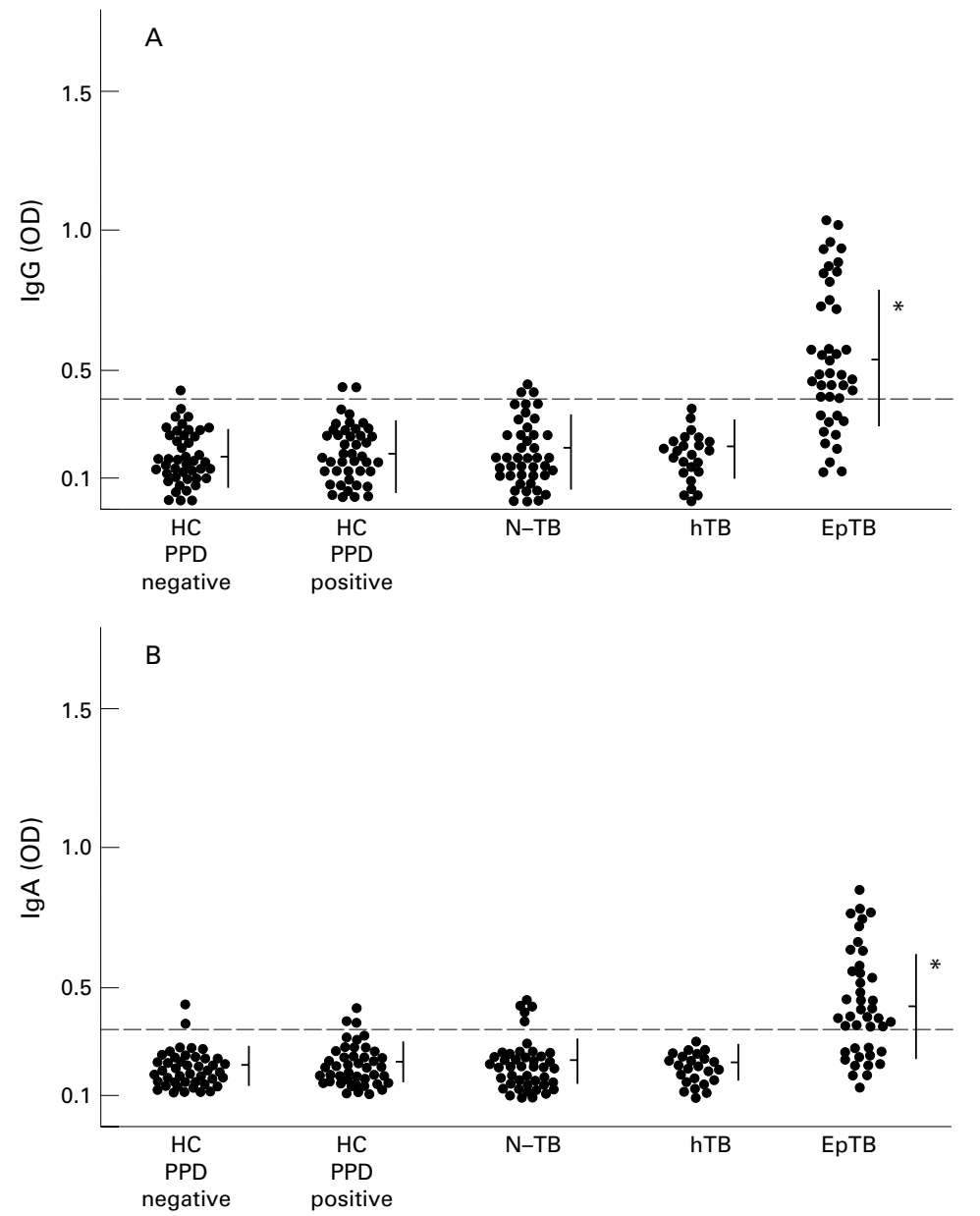

Figure 2 (A) Serum anti-A60 IgG and (B) IgA displayed for each diagnosis group. Each dot represents one subject. The broken lines represent the cut off values Vertical bars represent means (SD). $O D=$ optical density; $H C=$ healthy controls; $N-T B=$ non-tuberculous disease; $h T B=$ healed tuberculosis; EpTB = extrapulmonary tuberculosis. ${ }^{\star} p<0.0001$ versus HC PPD negative, HC PPD positive, $N-T B$, and $h T B$.

comparison, the Mantoux test was positive in 21 of the 42 cases. In all the subjects with healed tuberculosis $(n=24)$ the assay provided negative results. Positive test results were found in three of the 88 healthy subjects (one PPD negative and two PPD positive) and in three of 44 patients with non-tuberculous disease. Thus, overall sensitivity and specificity of the test were $73.8 \%$ and $96.1 \%$, respectively.

True positive rates (sensitivity) and false positive rates $(100 \%$ minus specificity) at different cut off values are shown in fig $1 \mathrm{~A}$.

$\operatorname{Ig} A$ test

Anti-A60 IgA levels in each diagnostic group are shown in fig $2 \mathrm{~B}$. Titres of IgA against A60 were significantly higher in patients with extrapulmonary tuberculosis than in control subjects $(\mathrm{p}<0.0001)$. When the cut off value was set at $0.321 \mathrm{OD}$, positive results were observed in 29 of 42 patients with extrapulmonary tuberculosis with a corresponding sensitivity of $69.0 \%$. All 24 patients with healed tuberculosis showed levels lower than the cut off value whereas in five of the 44 healthy controls (two PPD negative and three PPD positive) and in five of 44 patients with non-tuberculous disease the test was falsely positive. The specificity of the IgA assay was 93.6\%.

Figure 1B displays true positive rates and false positive rates at different cut off values.

Combined use of tests

When the results of both tests were considered the number of positive results in patients with extrapulmonary tuberculosis increased giving an overall sensitivity of $80.9 \%(+7.1$ vs IgG test, +11.9 vs $\operatorname{IgA}$ test). The number of false positive results increased to a lesser extent giving an overall specificity of $92.3 \%$ ( -3.8 vs IgG test, -1.3 vs IgA test). Eight of the 42 patients with extrapulmonary tuberculosis had negative results with both the IgG and the IgA assays; of the remaining 34 patients both tests were positive in 26 cases, whereas the IgG test alone was positive in five cases and the IgA test alone was positive in three cases.

\section{Discussion}

Searching for IgG against A60 is considered a useful diagnostic tool in pulmonary tuberculosis, with a reported sensitivity ranging between $78 \%$ and $94 \%$ by different authors. ${ }^{9}{ }^{10} \mathrm{We}$ recently reported that the detection of anti-A60 $\operatorname{IgA}$ is characterised by good sensitivity $(72.5 \%)$ and specificity (93.9\%) in pulmonary tuberculosis ${ }^{11}$; moreover, the combined use of the IgG and the IgA test increases overall diagnostic accuracy. ${ }^{11}$

In the present study patients with extrapulmonary tuberculosis showed significantly higher titres of both IgG and IgA against A60 compared with control groups. Both tests provided good characteristics of sensitivity and specificity. The combined use of the two tests allowed an increase in sensitivity more marked than the loss in specificity.

To our knowledge this is the first study to consider IgA serological testing against A60 in extrapulmonary tuberculosis undertaken in a western country. Gupta et $a l^{12}$ have recently reported a sensitivity of $73.8 \%$ and $68.4 \%$, respectively, for the anti-A60 IgG and IgA tests in an adult Indian population with extrapulmonary tuberculosis; the corresponding values of specificity were $92.4 \%$ and $93.4 \%$, respectively. The similarity of our results with those reported by Gupta et al suggests that some factors that may influence the diagnostic value of A60 based serological tests ${ }^{10}$ (different prevalence of tuberculosis, immunological status of the host, and phase and duration of disease before admission to hospital) play only a minor role.

In our study detection of IgG and $\operatorname{IgA}$ against $\mathrm{A} 60$ in patients with extrapulmonary tuberculosis was negative in a small percentage of cases. As already suggested, ${ }^{10}{ }^{11}$ this may be a result of immunosuppression due to the disease as well as to the presence of immune complexes that are quickly removed from the bloodstream.

Some false positive results of both tests occurred both among healthy volunteers (skin test positive as well as skin test negative) and patients with non-tuberculous diseases. This might be due either to subclinical infection of 
environmental non-tuberculous mycobacteria that also express A60, or to the presence in the host of commensal non-pathogenic mycobacteria. ${ }^{1011}$ The disregulation of the humoral immune response that occurs frequently in several diseases might be another cause of false positive results in patients with non-tuberculous disease. ${ }^{10}$

We did not include in our study BCG vaccinated healthy subjects. However, it has been reported that the BCG vaccination status does not affect the rate of false positive results of A60 based serological tests among healthy volunteers. ${ }^{10} 12$

In our series all subjects with a history of previously treated pulmonary or extrapulmonary tuberculosis showed negative results in both the IgG and the IgA tests. In patients treated for pulmonary tuberculosis the levels of IgG against $\mathrm{A} 60$ decreased following antituberculous chemotherapy. ${ }^{10}{ }^{15}$ The percentage of subjects with persistent positive serodiagnosis varies according to different reports ${ }^{12}$ : for comparison, Gupta et $a l^{12}$ reported that the percentage of positive anti-A60 IgG and IgA titres in patients with inactive pulmonary tuberculosis were $58.3 \%$ and $46.6 \%$, respectively. The differences observed by different authors are probably due to the varying time between the inclusion in the study and the end of antituberculous therapy. Our group of subjects with healed pulmonary or extrapulmonary tuberculosis consisted only of subjects treated several years before and this might explain the negativity of both tests observed in all the cases.

Our results of sensitivity and specificity were determined by using the cut off lines established according to the ROC curve method. Thus, our values of specificity might be further increased if the cut off lines were placed at higher values with an obvious loss in sensitivity (fig 1). As suggested for the serological test based on P90 antigen, ${ }^{8}$ we think that a positive result at a cut off level characterised by a high specificity may justify the starting of chemotherapy if tuberculosis is suspected while awaiting the results of culture; on the other hand, a positive result at a lower cut off value might be considered as one of the elements leading to a presumptive diagnosis of tuberculosis.

We conclude that the measurement of IgG and IgA against A60 may be considered a useful diagnostic tool in extrapulmonary tuberculosis, especially if molecular techniques are not available. Longitudinal studies are required to establish the diagnostic usefulness of A60 based serological testing in cases with negative results of microbiological and/or histological examinations.

The authors are grateful to Miss Elizabeth Kerford-Byrnes for technical assistance. This study was partly supported by the Italian Ministry of University (fondi MURST $60 \%$ ).

1 Weir MR, Thornton GF. Extrapulmonary tuberculosis: experience of a community hospital and review of the literature. Am f Med 1985;79:467-78.

2 Dolberg OF, Sclaeffer F, Greene VN, et al. Extrapulmonary tuberculosis in an immigrant society: clinical and demographic aspects of 92 cases. Rev Infect Dis 1991;13:177-9.

3 Kennedy DH, Fallon RJ. Tuberculous meningitis. $\mathcal{F} \mathrm{Am} \mathrm{Med}$ Assoc 1979;241:264-8.

4 Brisson-Noël A, Aznar C, Chureau C. Diagnosis of tuberculosis by DNA amplification in clinical practice evaluation. Lancet 1991;338:364-6.

5 Manjunath N, Shankar P, Rajan L, et al. Evaluation of polymerase chain reaction for the diagnosis of tuberculosis. Tubercle 1991;72:21-7.

6 Takahashi Y. Specific serum agglutination of kaolin particles sensitized with tubercle phosphatide and its clinical evaluation as a serodiagnostic test for tuberculosis. Am Rev Respir Dis 1962;85:708-19.

7 Benjamin RG, Daniel TM. Serodiagnosis of tuberculosis using ELISA (enzyme-linked immunosorbent assay) or antibody to $M$ tuberculosis antigen 5. Am Rev Respir Dis 1982;126:1013-6.

8 Alifano M, De Pascalis R, Sofia M, et al. Evaluation of IgAmediated humoral immune response against the mycobacterial antigen P-90 in diagnosis of pulmonary tuberculosis. Chest 1997;111:601-5.

9 Charpin D, Herbault H, Gevaudan MJ. Value of ELISA using A60 antigen in the diagnosis of active pulmonary tuberculosis. Am Rev Respir Dis 1990;142:380-4.

10 Cocito C. Properties of the mycobacterial antigen complex $\mathrm{A} 60$ and its applications to the diagnosis and prognosis of tuberculosis. Chest 1991;100:1687-93.

11 Alifano M, Sofia M, Mormile M, et al. IgA immune response against the mycobacterial antigen A60 in patients with activi.

12 Gupta S, Kumari S, Banwalikar JN, et al. Diagnostic utility of the estimation of mycobacterial antigen A60 specific immunoglobulins IgM, IgA and IgG in the sera of cases of adult human tuberculosis. Tubercle Lung Dis 1995;76:41824.

13 Metz CE. Basic principles of ROC analysis. Semin Nucl Med 1978;8:283-98.

14 Sackett DL, Haynes RB, Tugwell P. Clinical epidemiology. A basic science for clinical medicine. Boston: Little, Brown, 1985: 107-15.

15 Gevaudan MJ, Bollet C, Tissot-Dupont H, et al. Serological response of tuberculous patients to antigen 60 of BCG. Eur 7 Epidemiol 1992;8:666-76. 